\title{
EXPERIMENTAL STUDY OF WATER HAMMER PRESSURE SURGE
}

\author{
C. Bombardieri, T. Traudt, and C. Manfletti \\ Engine Transients Group, Institute of Space Propulsion \\ German Aerospace Center (DLR) \\ Hardthausen 74239, Germany
}

\begin{abstract}
During the start-up of the propulsion system of a satellite or spacecraft, the opening of the tank isolation valve will cause the propellant to flow into an evacuated feedline and slam against a closed thruster valve. This filling process, called priming, can cause severe pressure peaks that could lead to structural failure. In the case of monopropellants such as hydrazine, also, the risk of adiabatic compression detonation must be taken into account in the design of the feedline subsystem. The phenomenon of priming involves complex two-phase flow: the liquid entering the evacuated pipe undergoes flash evaporation creating a vapor cushion in front of the liquid that mixes with the residual inert gas in the line. Moreover, the dissolved pressurizing gas in the liquid will desorb making the priming process difficult to model. In order to study this phenomenon, a new test-bench has been built at DLR Lampoldshausen which allows fluid transient experiments in the same conditions as the operating space system. Tests are performed with water and ethanol at different conditions (tank pressure, vacuum level, pressurizing gas helium vs. nitrogen, etc.). The effect of the geometry is also investigated, comparing different test-elements such as straight, tees, and elbow pipes. The pressure profile is found to be dependent on the geometry and on the downstream conditions. The acoustic wave reflection caused by the pipe geometry and fluid dynamic effects such as the aforementioned desorption and flash evaporation induce a complex pressure profile of the first pressure peak. Finally, numerical simulations of the priming process are performed by means of EcosimPro software in conjunction with European Space Propulsion System Simulation (ESPSS) libraries and results are compared with experiments.
\end{abstract}

\section{INTRODUCTION}

For safety reasons, the propellant feedlines of a satellite or spacecraft are usually evacuated prior to launch. Once in orbit, the start-up of the propulsion system 
of the satellite involves the opening of the tank isolation valve that will cause the propellant to flow into the evacuated feedline and slam against a closed thruster valve. This filling process, called priming, can cause severe pressure peaks that can lead to structural failure. In the case of monopropellants such as hydrazine, also, the risk of adiabatic compression detonation must be taken into account in the design of the feedline subsystem. The hazard of the adiabatic compression detonation was experimentally proved by Bunker et al. [1]. They demonstrated that the explosive events observed in hydrazine experiments were caused by chemical reactions of liquid hydrazine (exothermic decomposition) not induced by water-hammer effects but rather due to the rapid compression of the noncondensable gas present in the line. Hydrazine detonation accidentally occurred during the testing of the satellite propulsion system for the ISPM (International Solar Polar Mission) [2] which led to the destruction of the entire test-bench. According to the authors, the cause was (probably) a local high temperature along the pipe which caused the detonation of hydrazine.

To prevent this potential hazard, the solution is to slow down the flow through the use of a flow restriction device (venturi $[3,4]$ or orifice $[5,6]$ ) or by using the gas cushion effect of a prefilled inert gas in the line.

The phenomenon of priming involves complex two-phase flow: the liquid entering the evacuated pipe undergoes flash evaporation creating a vapor cushion in front of the liquid that mixes with the residual inert gas, usually helium. Moreover, the dissolved pressurizing gas in the liquid will desorb making the priming process difficult to model numerically due to the lack of understanding of these physical processes. Numerical codes available today for fluid transient calculations can give accurate predictions for single-phase water hammer, but they still need improvements while simulating two-phase/two-component flows. The creation of an extensive experimental database is, therefore, necessary for the validation of these numerical tools. Although the aerospace industries perform water hammer tests as a part of the qualification campaign of their propulsion subsystems, basic research in the field is rather limited.

Gibec and Maisonneuve [7] performed water hammer experiments with real propellants, namely, monomethyl hydrazine (MMH), nitrogen tetroxide (NTO), and hydrazine, for different pipe geometries including straight, bend, elbow, and tee pipes. They hypothesized that phenomena such as cavitation, pipe deformation, and vapor pressure may interfere with the water hammer. Lecourt and Steelant [8] performed several tests with ethanol, acetaldehyde, and MMH for straight and bend pipes. They observed a surprising multiple-step evolution of the first pressure peak and provided a possible explanation. They also demonstrated that ethanol can be used as a replacement fluid instead of toxic MMH. Lema and Steelant [9] investigated the effect of a fully saturated liquid compared to a deareated one in vacuum conditions. Test results showed that for the saturated liquid, the pressure peak is slightly smaller due to the desorption of the dissolved gas which acts as a cushion in front of the liquid. 
This paper presents the experimental results of priming tests run with different geometry setups. Test fluids are water and ethanol. In addition, numerical simulations are also presented and results compared with experiments.

\section{EXPERIMENTAL SETUP}

\subsection{Test Bench Description}

To investigate and gain detailed insight into the filling process of feedlines, a new test bench has been built at DLR Lampoldshausen. The test bench features a 80-liter run tank pressurized up to 50 bar, a flexible pressurization system (gaseous nitrogen $\left(\mathrm{GN}_{2}\right)$ or gaseous helium $(\mathrm{GHe})$ as a pressurizing gas) as well as a modular test section with its own conditioning system. This modularity ensures that the test bench is not limited to one test section but it can reproduce a real spacecraft feedline system geometry. Conditioning of the test section can be either done via evacuation or pressurization. The test bench is equipped with a fast opening valve (FOV), pneumatically actuated, mounted on a rigid support to limit vibrations during the opening (Fig. 1). Its opening time is only $6 \mathrm{~ms}$. As reported in previous papers [8-10], the valve opening time should be faster than the travel time of the liquid front to the dead-end. The impact time, as will be shown later, is in the range 140-150 ms.

The valve opening transient is an important boundary condition for numerical validation. A requirement for the experimental setup was, therefore, to have a position measurement sensor not only to ensure reproducibility of the valve

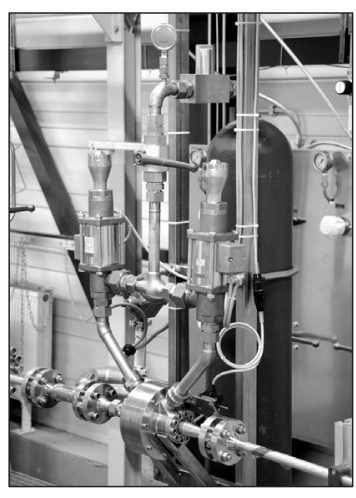

(a)

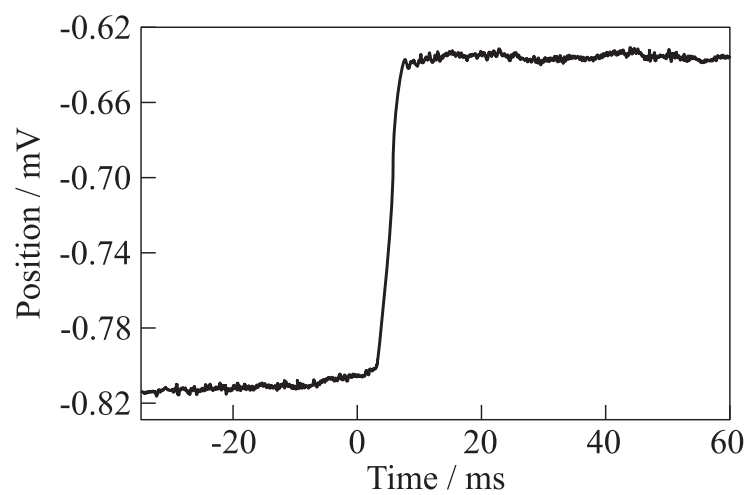

(b)

Figure 1 Fast opening valve pneumatically actuated $(a)$ and its opening profile $(b)$ 
opening transient but also to provide the necessary input for numerical simulations. The valve features a position encoder and its opening profile (an example in Fig. 1) was perfectly reproducible over all the tests performed.

\subsubsection{Geometries for priming tests}

Schematic of the test facility is shown in Fig. 2. The geometry of the test-element is a 2000-millimeter straight stainless steel pipe (1.4541, AISI 321) with a relative large outer diameter $(3 / 4$ in or $19.05 \mathrm{~mm})$ in order to examine high mass flow rate that is typical of spacecraft feedlines like the European Space Agency (ESA) Automatic Transfer Vehicle (ATV).

The wall thickness of the test section is $1.25 \mathrm{~mm}$ (ID $16.56 \mathrm{~mm}$ ). At 5 points, it is fixed onto a support structure by means of clamps to limit its movements. The test section is mounted with a downward slope of about $1^{\circ}$ to facilitate the purging procedure. The upstream segment, from the tank to the valve, is a $22 \times 1.5$-millimeter straight stainless steel pipe with a tee piece inserted $550 \mathrm{~mm}$ downstream the tank to allow purging and evacuation. The detailed geometry of the test bench is given in Table 1 .

When different configurations are to be tested, the test-element is removed and replaced by the desired geometry. In the experiments presented in this paper, four setups are tested: straight, tees (T, T2), and elbow (L) configurations. The part upstream of the FOV is kept the same. The T geometry has a 1000-millimeter straight pipe followed by two branches of $500 \mathrm{~mm}$ each, in a left/right arrangement. Similarly, the T2 geometry has a 1000-millimeter straight pipe but with asymmetrical branches, respectively, 1000 and $500 \mathrm{~mm}$.

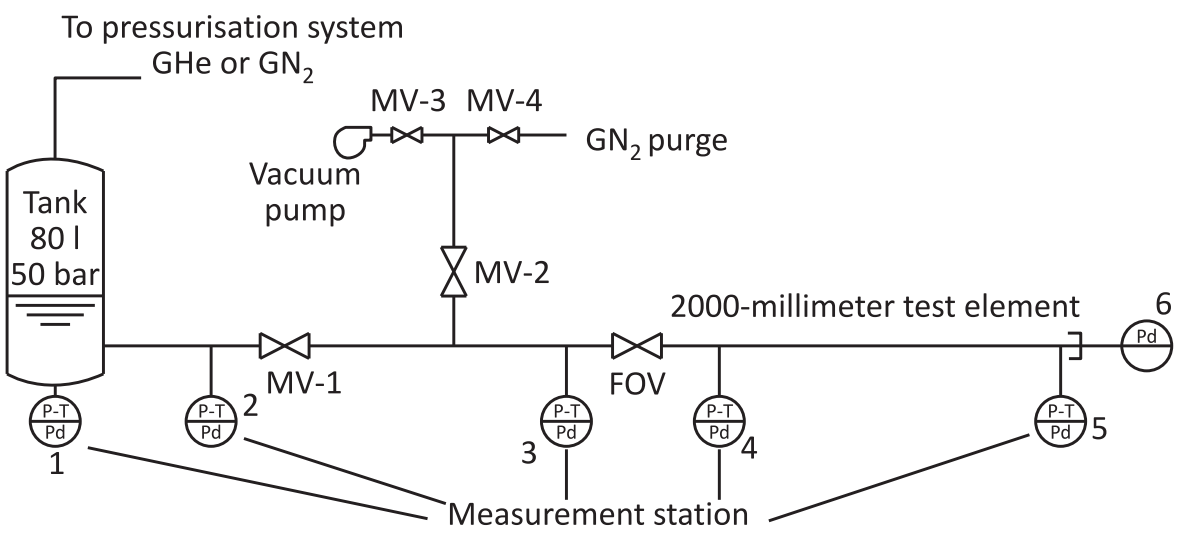

Figure 2 Schematics of M3.5 fluid transient test facility at DLR Lampoldshausen. The 2000-millimeter straight test section is depicted 
Table 1 Dimensions of the test-bench

\begin{tabular}{lc}
\hline Description & Value \\
\hline Upstream pipe tank-FOV & $1023 \mathrm{~mm}$ \\
Position of T-branch (MV-2) from tank & $550 \mathrm{~mm}$ \\
Branch length to MV-2 & $130 \mathrm{~mm}$ \\
FOV seat & $16 \mathrm{~mm}$ \\
Test-section length & $2000 \mathrm{~mm}$ \\
Test-section outer diameter $\times$ wall thickness & $19.05 \times 1.25 \mathrm{~mm}$ \\
\hline
\end{tabular}

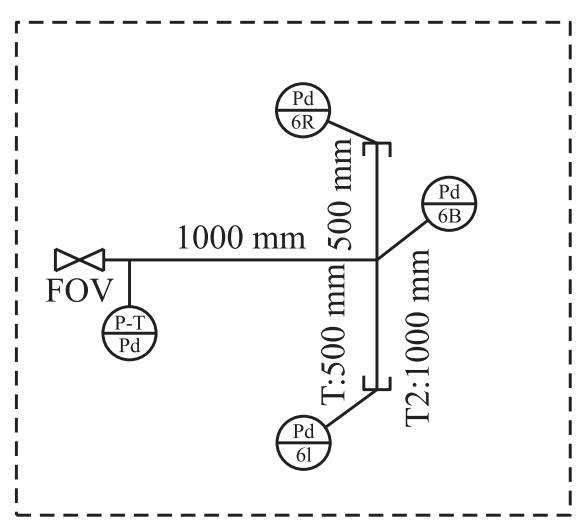

(a)

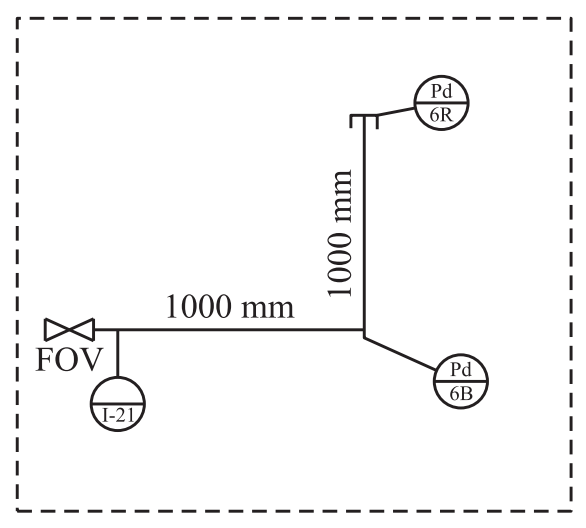

(b)

Figure 3 Configuration of the T/T2 $(a)$ and L (b) geometries used

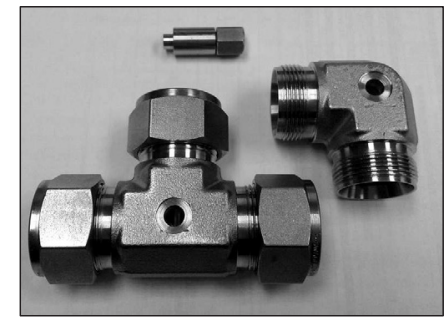

(a)

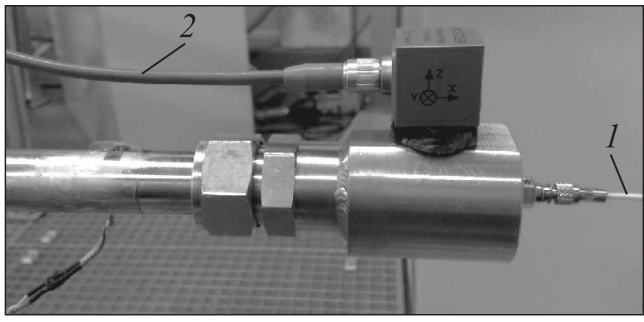

(b)

Figure 4 Details of the $\mathrm{T}$ and $\mathrm{L}$ connectors with the insert hole for the pressure sensor $(a)$; and the dead-end measurement piece with the pressure sensor (1) and an accelerometer $(2)(b)$ 
The final geometry is an elbow (L), a 1000-millimeter straight pipe followed by another 1000-millimeter straight pipe at $90^{\circ}$. The $\mathrm{T}$ and $\mathrm{L}$ setups with their measurement stations are shown in Fig. 3. The $\mathrm{T}$ and $\mathrm{L}$ configurations have been sized so that the filling volume is the same as the 2000-millimeter straight pipe in order to quantitatively compare the pressure peak and the impact time. The different branches are connected via Swagelok screw connectors shown in Fig. 4.

\subsubsection{Sensors}

Measurement of pressure and temperature is performed at 6 different stations as shown in Fig. 2. Each measurement station consists of three transducers: one thermocouple type k, 1-kilohertz sampling rate; one absolute piezoresistive pressure sensor type 4043A200 from Kistler, 10-kilohertz sampling rate; and one dynamic piezoelectric pressure sensor type 601A from Kistler, 150-kilohertz sampling rate.

To avoid aliasing and high-frequency noise, the filter of the dynamic pressure sensors has been set to $30 \mathrm{kHz}$.

Sensors are screwed in a 20-millimeter thick disk with the same inner diameter of the pipe to avoid flow disturbances. Dynamic pressure sensors (5.5-millimeter diameter) and thermocouple are flush mounted, while the absolute pressure sensor is $2 \mathrm{~mm}$ beneath the surface through a 1-millimeter hole. The measurement stations are located as follows:

- pos. 1: at the tank;

- pos. 2: $250 \mathrm{~mm}$ downstream of the tank;

- pos. 3: $318 \mathrm{~mm}$ upstream of the FOV;

- pos. 4: $160 \mathrm{~mm}$ downstream of the FOV;

- pos. 5: $1990 \mathrm{~mm}$ downstream of the FOV (10 mm from the dead-end); and

- pos. 6: at the dead-end (only dynamic pressure).

In the case of $\mathrm{T}$ and $\mathrm{L}$ geometries, an additional dynamic pressure sensor is placed at the junction (Fig. 4) named Pd-6B. For T and T2 configurations, the dead-end of the second branch is also provided with a dynamic pressure sensor named Pd-6L. Figure 3 shows the positions of these additional sensors.

\section{$2.2 \quad$ Test Procedure}

Before each test, the downstream test-section is purged with a $\mathrm{GN}_{2}$ flow by opening MV-4 and MV-2 (see Fig. 1) and unscrewing the measurement module at 
the test-element end. After this operation, the test section is evacuated by means of a vacuum pump (MV-3 open) to the desired low pressure level. The FOV and $\mathrm{MV}-2$ are then closed and MV-1 is opened to manually prime the upstream pipe. At this point, automatic operations are performed by the controlling software: the tank pressure is set at a given value, the trigger command for data acquisition is given $(-500 \mathrm{~ms})$, and FOV opens (time: $0 \mathrm{~ms}$ ). Data are recorded for $4 \mathrm{~s}$.

\subsection{Test Matrix}

In the case of the 2000-millimeter straight test section, tests with different pressures in the test-element were performed, while the tank pressure was kept at 20 bar. The pressure in the test-element, or line pressure, for water and ethanol has been set so that the same ratio with respect to their saturation pressure is kept. The vacuum pressure levels are shown in Table 2. Then, the different geometries are tested at vacuum condition only. The residual gas in the line is $\mathrm{GN}_{2}$.

Table 2 Test-matrix: Line pressure for water and ethanol for the different tested geometries $\left(P_{\text {tank }}=20\right.$ bar $)$

\begin{tabular}{lcc}
\hline Geometry & Water, $P_{\text {sat }}=19.2 \mathrm{mbar}$ & Ethanol, $P_{\text {sat }}=40.6 \mathrm{mbar}$ \\
\hline Straight & $300-100-50-10 \mathrm{mbar}$ & $400-200-100-20 \mathrm{mbar}$ \\
$\mathrm{T}$ & $<10 \mathrm{mbar}$ & $<20 \mathrm{mbar}$ \\
$\mathrm{T} 2$ & $<10 \mathrm{mbar}$ & - \\
$\mathrm{L}$ & $<10 \mathrm{mbar}$ & $<20 \mathrm{mbar}$ \\
\hline
\end{tabular}

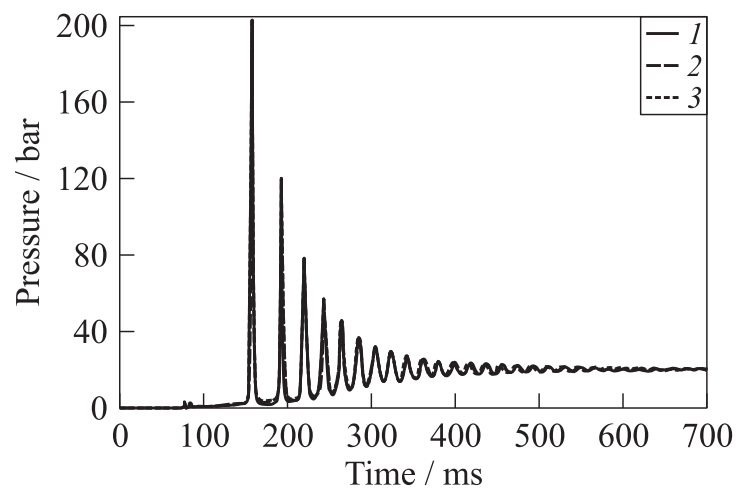

Figure 5 Example of reproducibility; pressure signal at dead-end with straight pipe, fluid is water: 1 - test \#01;2 - test \#02; and 3 - test \#03 
Water is pressurized with $\mathrm{GN}_{2}$ while for ethanol, GHe is used. Tests are repeated three times for each test condition to examine reproducibility. Figure 5 shows an example of the reproducibility achieved. The measured pressure peak difference between the three tests is less than $1.5 \%$. From an experimental point of view, reproducibility of ethanol tests is better than that obtained in water tests.

\section{TEST RESULTS}

The target of the experimental campaign is to reproduce the filling process occurring in a spacecraft feedline system and, in particular, to investigate the effect of different geometries on the water hammer pressure profile. These experiments will also provide a database to validate numerical simulations. In this paper, only the results of tests at vacuum condition (10 mbar for water and 20 mbar for ethanol) are presented and discussed.

The water hammer pressure peak can be estimated with the Joukowsky's equation*:

$$
P=\rho c V
$$

where $\rho$ is the fluid density; $c$ is the speed of sound in the fluid; and $V$ is the impact velocity at the dead-end. As reported in previous papers $[3,11,12]$, an analytical model based on the rigid liquid column theory can be applied to the priming process of a satellite system to predict the impact velocity of the propellant with satisfactory results (error within $6 \%$ with respect to the experimental data). The model considers the liquid as an incompressible slug in unsteady motion. The main issue is to correctly take the friction factor into account, since the results are strongly sensitive to this value [12]. Velocity $V$ can be expressed in terms of pressure difference and density according to Bernoulli's equation:

$$
V \approx \sqrt{\frac{P_{\text {tank }}-P_{\text {line }}}{\rho}} .
$$

Therefore, at the same pressure conditions, the water hammer pressure peak of two different fluids can be related as follows:

$$
\frac{P_{1}}{P_{2}}=\frac{\sqrt{\rho_{1}} c_{1}}{\sqrt{\rho_{2}} c_{2}} .
$$

Evaluating Eq. (2) for ethanol $\left(c=1124 \mathrm{~m} / \mathrm{s}\right.$ and $\left.\rho=796 \mathrm{~kg} / \mathrm{m}^{3}\right)$ and water $\left(c=1361 \mathrm{~m} / \mathrm{s}\right.$ and $\left.\rho=1000 \mathrm{~kg} / \mathrm{m}^{3}\right)$ gives a theoretical water hammer pressure

*Joukowsky's equation gives actually the overpressure $\Delta P=\rho c \Delta V$. However, in these experiments, the initial pressure is zero being (almost) vacuum. 
ratio $P_{\text {eth }} / P_{w}=0.74$. The speed of sound is calculated taking the elasticity of the pipe material into account according to the Korteweg's formula:

$$
c^{2}=\frac{c_{f}^{2}}{1+\left(E_{f} / E_{w}\right)(D / t) \mu}
$$

where $c_{f}$ is the unconfined fluid speed of sound; $E_{f}$ is the fluid compressibility modulus; $E_{w}$ is the elasticity modulus of the material; $D$ is the pipe diameter; $t$ is the wall thickness; and $\mu$ is the coefficient depending on the pipe support boundary condition.

\subsection{Straight pipe}

Test results obtained with the 2000-millimeter straight pipe are shown in Fig. 6 for water and ethanol. The maximum pressure peak is 202 and 139 bar, respectively. The pressure ratio ethanol/water is, therefore, 0.69 , close to the theoretical value of 0.74 . One should also consider that the viscosities of the two fluids are different and that affects the final impact velocity.

The attenuation of the wave is very similar. With respect to the first pressure peak, the second pressure peak is, in fact, $58.4 \%$ for water and $59 \%$ for ethanol (118 and 82 bar). For both fluids, the pressure is more or less damped off $300 \mathrm{~ms}$ after the main pressure peak.

The results of the straight pipe tests will be used to validate the numerical simulations.

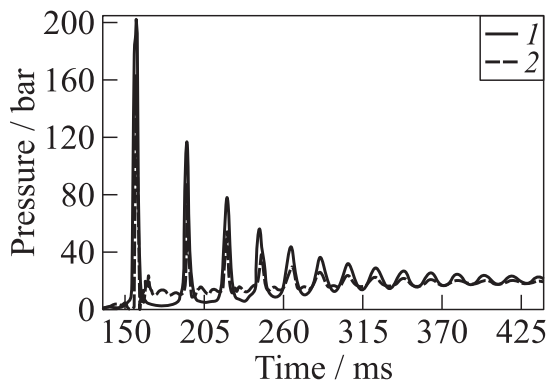

(a)

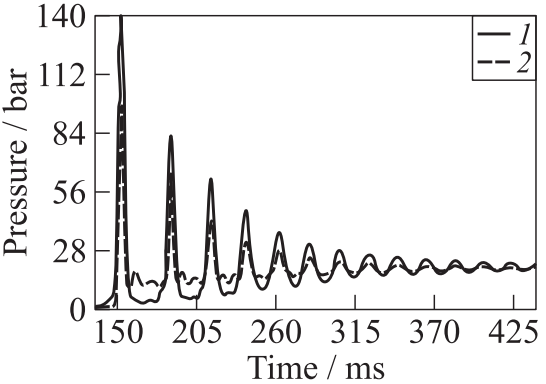

(b)

Figure 6 Pressure evolution for water $(a)$ and ethanol $(b)$ with the 2000-millimeter straight test-element at a vacuum line pressure: 1 - dead-end (PD-6); and 2 - after valve (PD-4) 


\subsection{Tees and elbow geometries}

The pressure history at the dead-end for different tested geometries is shown in Fig. 7. Table 3 summarizes the pressure peaks obtained in the different geometries. As expected, the pressure peaks for $\mathrm{T}$ and $\mathrm{L}$ configurations are smaller than the straight pipe case due to the momentum loss introduced by the connector. The attenuation of the pressure wave is more pronounced in the case of the $\mathrm{T}$ configuration for both water and ethanol.

In the case of water and L configuration, the first pressure peak shows a double spike, with the first spike at $165 \mathrm{~ms}$ and the second spike at $172 \mathrm{~ms}$. This is not present in the case of ethanol. In an attempt to better understand the origin of it, an additional test-campaign has been run using GHe instead of $\mathrm{GN}_{2}$ as the pressurizing gas for water. Preliminary results indicate that this double spike is due to a combined effect of the dissolved gas $\mathrm{GN}_{2}$ in water and the concentrated pressure loss at the L-piece. With GHe tests, this phenomenon does not occur as GHe is much less dissolvable in water than $\mathrm{GN}_{2}$. The authors hypothesize that the L-piece enhances the gas desorption from the liquid and causes the separation of a liquid slug that precede the main flow and hit the

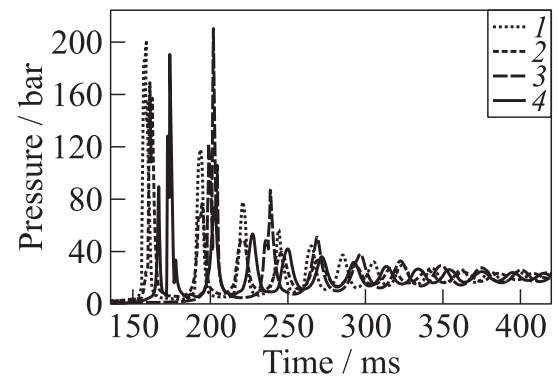

(a)

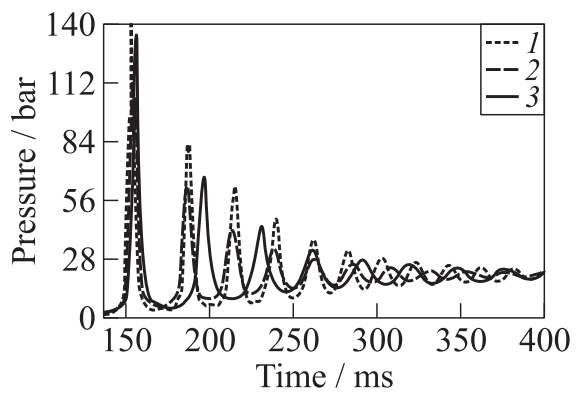

(b)

Figure 7 Pressure profile for different tested geometries $(1-$ straight; $2-\mathrm{T} ; 3-$ $\mathrm{T} 2$; and $4-\mathrm{L})$ for water $(a)$ and ethanol $(b)$

Table 3 Pressure peaks for different tested geometries $\left(P_{\text {tank }}=20\right.$ bar $)$

\begin{tabular}{lcc}
\hline Geometry & Water, $P_{\text {line }}=10$ mbar & Ethanol, $P_{\text {line }}=20$ mbar \\
\hline Straight & $201.9 \mathrm{bar}$ & $139.4 \mathrm{bar}$ \\
$\mathrm{T}$ & $168.8 \mathrm{bar}$ & $112.5 \mathrm{bar}$ \\
$\mathrm{T} 2$ & $210.0 \mathrm{bar}$ & - \\
$\mathrm{L}$ & $190.8 \mathrm{bar}$ & $134.4 \mathrm{bar}$ \\
\hline
\end{tabular}


dead-end (first spike), followed a few milliseconds later by the main flow. When injected in vacuum, the liquid undergoes flash boiling and, in addition, starts desorbing the dissolved gas. Additional tests are in progress to validate this hypothesis.

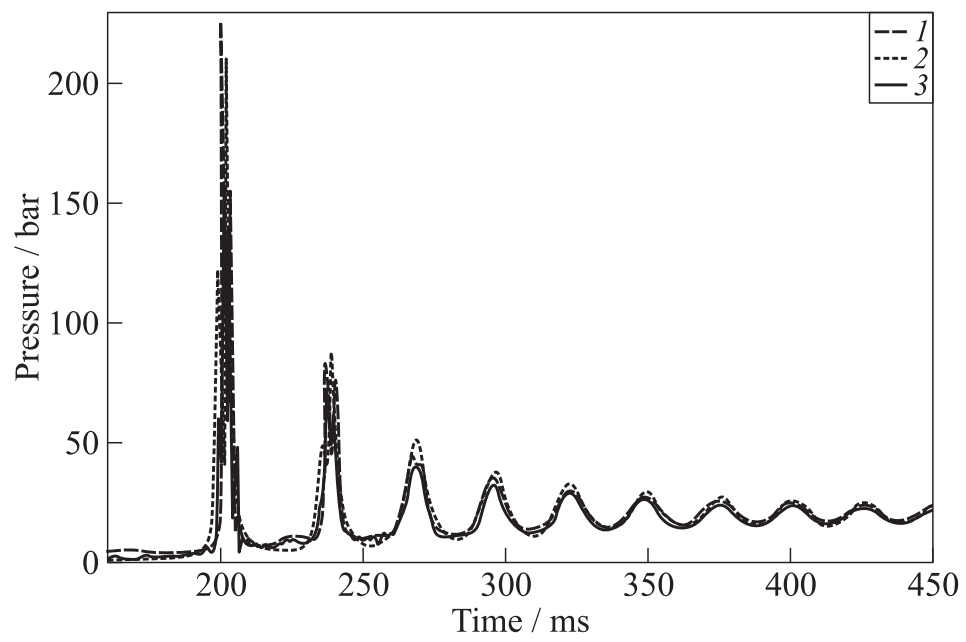

(a)

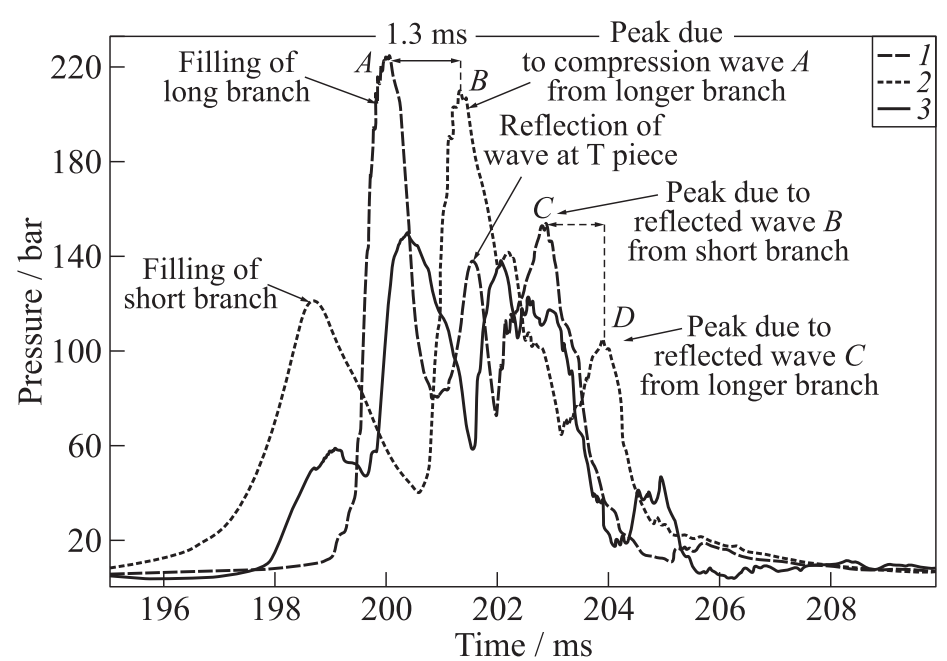

(b)

Figure 8 Pressure profiles at the dead-ends for the T2 geometry: $1-\mathrm{PD}-6 \mathrm{~L}$ is the pressure sensor at the longer branch dead-end; $2-\mathrm{PD}-6 \mathrm{R}$ is the sensor at the shorter branch dead-end; and $3-\mathrm{PD}-6 \mathrm{~B}$ is the pressure sensor at the T-connector 
In the case of the $\mathrm{T}$ geometry, the pressure peaks in the two branches deadends are identical ( $<1$ bar difference) and their impact time is practically the same: the maximum time delay between the peaks in the two branches is $0.2 \mathrm{~ms}$. The effect of the pressure wave reflection is visible in the case of asymmetrical branches (T2). Figure 8 depicts the pressure evolution at both dead-ends and at the tee junction for the T2 geometry. The profile of the pressure peak at both ends is the result of multiple reflections of the pressure wave traveling from one end to the other. First, the filling of the branch generates the first pressure peak, occurring sooner for the shorter branch. The higher pressure peak (225 bar, PD$6 \mathrm{~L})$ takes place in the longer branch because it is the last cavity to be filled with the liquid. This causes a compression wave that propagates to the shorter branch inducing a second spike (210 bar, PD-6R) which, in turn, causes a spike on the longer branch.

The time delay between the two peaks is $1.3 \mathrm{~ms}$. Considering the distance between the two sensors $(1500 \mathrm{~mm})$, this gives a speed of sound of $1154 \mathrm{~m} / \mathrm{s}$. This value is $15 \%$ smaller than the theoretical value of $1361 \mathrm{~m} / \mathrm{s}$ for water: this is due to the amount of residual gas in the line that reduces the speed of sound.

The main wave moves subsequently upstream towards the tank and the pressure in the test-element ends drops to about 5 bar. When the main pressure wave moves again downstream, the mutual interference of the reflections is still present in the second pressure peak (at about $240 \mathrm{~ms}$ ). Now, the shorter branch has a slightly higher pressure of 86 bar compared to 82 bar of the longer branch. From the forth peak on $t>290 \mathrm{~ms}$, the effect of the acoustic reflections is no longer detectable in the pressure profile.

In future, the experiments will be run with different branch lengths and also with a different arrangement of the T-piece, e.g., bottom/side instead of left/right.

\section{NUMERICAL SIMULATIONS}

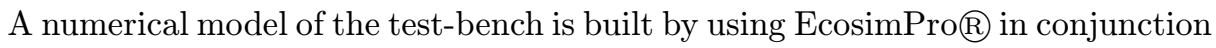
with the ESPSS library, an ESA-developed tool capable of one-dimensional, twophase flow transient simulations. The model schematics used for the simulation is shown in Fig. 9.

For the simulation, the straight 2000-millimeter test-element is modeled and results with water are used. As described in the present authors' previous work [12], the friction factor is to be increased in order not to overpredict the pressure peak. The increase of the friction factor is physically motivated by the unsteady conditions of the flow. Changes occurring in the velocity profile during acceleration of the flow produce varying shear stress at the wall. Due to this 


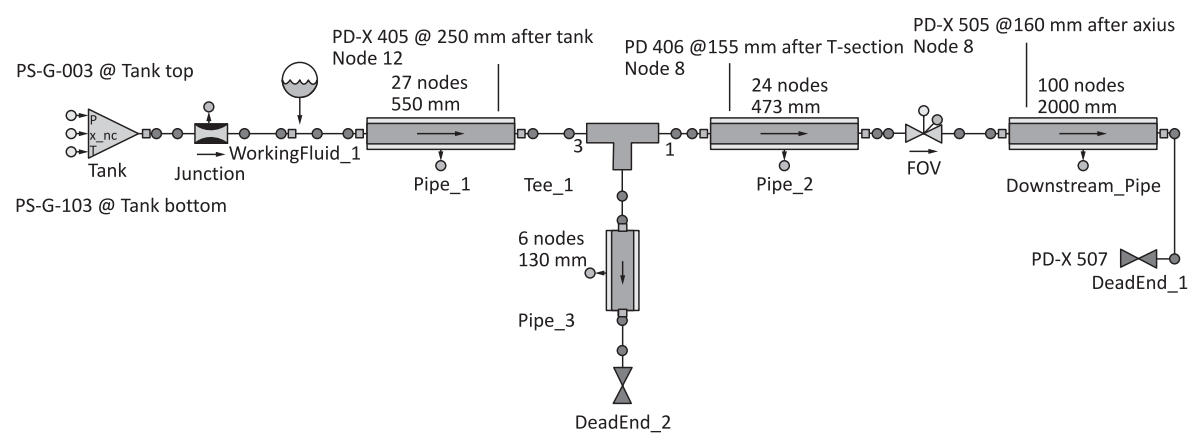

Figure 9 EcosimPro/ESPSS model of the test-bench used for the simulation

varying shear stress, the viscous boundary layer is not established and a higher velocity gradient is found at the wall than would be expected for steady flow. Because of the higher velocity gradient at the wall, a greater friction coefficient should be used. Empirically, a multiplier value of 3 for the friction factor is set in order to better agree with the experimental data.

First, a grid sensitivity study is carried out. The test-element is modeled with an increasing number of nodes, respectively, 50, 100, and 200 nodes. While the first pressure peak is identical in each case (Fig. 10), the frequency and the attenuation of the wave strongly depend on the number of nodes. The arrival time of the first peak is $\sim 11 \mathrm{~ms}$ faster than the experimental value (note that in Fig. 10, the experimental curve has been shifted to match the pressure peaks

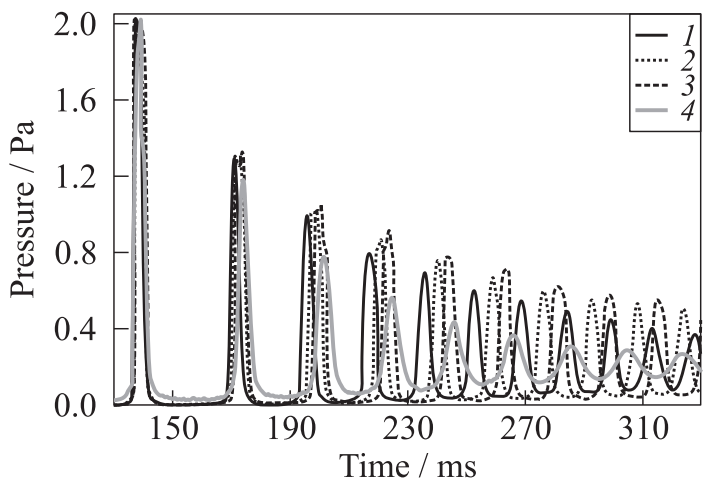

Figure 10 Comparison between numerical results $(1-50$ nodes; $2-100$; and $3-$ 200 nodes) and experimental data (4) for water, straight pipe, 10 mbar in the testelement. The experimental data have been shifted in $-11 \mathrm{~ms}$ to make the comparison easier 
to allow a better comparison). The predicted value of the first pressure peak matches the experimental one: it would suggest that the impact velocity at the dead-end is also the same, with respect to the Joukowsky's equation (see Eq. (1)). Nevertheless, the friction factor has been adjusted to artificially match the pressure peak but that also affects the acceleration history of the flow. Two different acceleration profiles can lead to the same final velocity yet with different impact time. That could be easily seen by considering the final velocity as the definite integral of the acceleration.

Increasing the number of nodes leads to a decrease in the frequency and to a less damped wave. The experimental frequency calculated after the 4 th peak (to avoid the effect of the column separation at the dead-end) is $51 \mathrm{~Hz}$, while the numerical one is $60 \mathrm{~Hz}$ in the case of 200 nodes. The reason behind this disagreement is the difficulty of calculating the speed of sound in a two-phase flow. The presence of gas decreases the speed of sound in a nonlinear way. Since the real fluid, when injected in vacuum, desorbs the dissolved pressurizing gas, an extra amount of gas is added to the residual one in the line (10 mbar of $\mathrm{GN}_{2}$ ). This additional amount further decreases the speed of sound $c$ and, thus, the frequency $f$, as $f=c /(4 L)$. In the numerical model, the desorption of gas from the liquid is not taken into account and, therefore, the numerical frequency is higher than the experimental one.

The wave attenuation is also underestimated: the experimental pressure signal is more damped than the numerical prediction. This is due to three main effects:

(1) the aforementioned additional released gas which further attenuates the pressure wave;

(2) the friction factor which, as already explained, is modeled in ESPSS only for steady flow conditions; and

(3) the structural vibrations absorbing some energy.

The implementation of an unsteady friction model will be addressed in future work as well as a more detailed experimental investigation on the fluid-structure interactions.

\section{CONCLUDING REMARKS}

At DLR Lampoldshausen, a new test facility to investigate the filling process occurring in spacecraft and satellite feedlines has been built. Priming tests in evacuated pipelines have been performed with water and ethanol, the latter being the best replacement fluid for the toxic hydrazine. The theoretical correlation based on the fluid properties to relate the water hammer pressure peak between 
two fluids proves to give a satisfactory result when compared against the test data.

Different geometries have been tested, namely, straight pipe, elbow, and tees. The use of junctions such as elbow and tees causes not only a reduction of the pressure peak with respect to the straight pipe, but also a different shape in the pressure history. In the case of the elbow test-section, the authors hypothesize that the desorption of the dissolved gas has a strong influence on the pressure peak causing a double-spike profile. Preliminary results confirmed the influence of the pressurizing gas: when helium is used instead of nitrogen, the pressure profile is different. Further tests are in progress to investigate this aspect.

The tee setup with asymmetrical branches shows the effect of the mutual interference of the acoustic reflection resulting from the two dead-ends. The pressure peak at one dead-one causes a wave reflection that affects the pressure profile at the opposite end and this can lead to higher pressure peaks.

Numerical simulations have been performed by means of EcosimPro in conjunction with library ESPSS. A grid sensitivity study showed that the frequency and the wave attenuation are dependent on the number of nodes, while the first pressure peak is not affected. The calculated frequency is higher than the experimental one due to the difficulty in calculating the speed of sound for a two-phase flow. The lack of a model for the desorption of gas from the liquid leads to underestimation of the amount of gas and, therefore, induces a higher speed of sound and, consequently, higher wave frequencies. For the same reason, the attenuation of the pressure wave is also not well predicted, where the experimental pressure signal is more damped than the predicted one. The inadequacy of the steady friction model when applied to unsteady flow contributes also to the mismatching. In unsteady conditions, the friction factor is higher than in steady flow which results in a more damped pressure wave. Future simulations will include the implementation of an unsteady friction model, while future experimental work will focus on fluid-structure interactions.

\section{REFERENCES}

1. Bunker, R. L., D. L. Baker, and J.H. S. Lee. 1991. Explosive decomposition of hydrazine by rapid compression of a gas volume. Dynamics of detonations and explosions: Detonations. Eds. J.-C. Leyer, A. A. Borisov, A. L. Kuhl, and W. A. Sirignano. Progress in astronautics and aeronautics ser. 133:325-341.

2. Hutchinson, F. E., and H. D. Schmitz. 1984. Adiabatic compression phenomena in hydrazine propulsion systems. 20th AIAA/SAE/ASME Joint Propulsion Conference. Cincinnati, $\mathrm{OH}$.

3. Hearn, H. C. 2005. Development and application of a priming surge analysis methodology. 41st AIAA/ASME/SAE/ASEE Joint Propulsion Conference \& EXhibit. 
4. Scroggins, A.R. 2012. A streamlined approach to venturi sizing AIAA Paper No. 2012-4028.

5. Chang, Y. J., and K.D. Park. 2000. Pressure surge analysis and reduction in the KOMPSAT propellant feed system. KORUS 2000 Proceedings: 4th Korea-Russia Symposium (International) on Science and Technology.

6. Morgan, M. J. 2004. Pressure transient characterization test for Star-2 propulsion system fuel manifold. 40th AIAA/ASME/SAE/ASEE Joint Propulsion Conference and Exhibit.

7. Gibek, I., and Y. Maisonneuve. 2005. Waterhammer tests with real propellants. AIAA Paper No. 2005-4081.

8. Lecourt, R., and J. Steelant. 2007. Experimental investigation of waterhammer in simplified basic pipes of satellite propulsion systems. J. Propul. Power 23:12141224.

9. Lema, M., and J. Steelant. 2012. Experimental characterization of the priming phase using a propellant line mock-up. Space Propulsion Conference. Bordeaux, France.

10. Lin, T. Y., and D. Baker. 1995. Analysis and testing of propellant feed system priming process. J. Propul. Power 11:505-512.

11. Yaggy, K. L. 1984. Analysis of propellant flow into evacuated and pressurized lines. AIAA Paper No. 1984-1346.

12. Bombardieri, C., T. Traudt, and C. Manfletti. 2014. Experimental and numerical analysis of water hammer during the filling process of pipelines. Space Propulsion Conference. Cologne, Germany. 